\title{
Genetic determinants of variable metabolism have little impact on the clinical use of leading antipsychotics in the CATIE study
}

Iris Grossman, $P h D^{1}$, Patrick F. Sullivan, $M D^{2}$, Nicole Walley, BSc ${ }^{1}$, Youfang Liu, MSc ${ }^{3}$, Jeffrey R. Dawson, BSc ${ }^{1}$, Curtis Gumbs, BSc ${ }^{1}$, Andrea Gaedigk, PhD ${ }^{4}$, J. Steven Leeder, PharmD, PhD ${ }^{4}$, Joseph P. McEvoy, $M D^{5,6}$, Michael E. Weale, $P h D^{1}$, and David B. Goldstein, $P h D^{1}$

\begin{abstract}
Purpose: To evaluate systematically in real clinical settings whether functional genetic variations in drug metabolizing enzymes influence optimized doses, efficacy, and safety of antipsychotic medications. Methods: DNA was collected from 750 patients with chronic schizophrenia treated with five antipsychotic drugs (olanzapine, quetiapine, risperidone, ziprasidone, and perphenazine) as part of the Clinical Antipsychotic Trials of Intervention Effectiveness study. Doses for each of the medicines were optimized to 1, 2, 3, or $4 \times$ units in identically appearing capsules in a double-blind design. We analyzed 25 known functional genetic variants in the major and minor metabolizing enzymes for each medication. These variants were tested for association with optimized dose and other relevant clinical outcomes. Results: None of the tested variants showed a nominally significant main effect in association with any of the tested phenotypes in European-Americans, African-Americans, or all patients. Even after accounting for potential covariates, no genetic variant was found to be associated with dosing, efficacy, overall tolerability, or tardive dyskinesia. Conclusion: There are no strong associations between common functional genetic variants in drug metabolizing enzymes and dosing, safety, or efficacy of leading antipsychotics, strongly suggesting merely modest effects on the use of these medicines in most patients in typical clinical settings. Genet
\end{abstract} Med 2008:10(10):720-729.

Key Words: pharmacogenetics, CYP 450, drug metabolizing enzymes, antipsychotics, personalized medicine

Drug metabolizing enzymes (DMEs) have been a key focus of pharmacogenetics since its inception in the 1950s. ${ }^{1,2} \mathrm{~A}$ large body of evidence collected over the proceeding decades by various independent groups shows a direct impact of functional

\footnotetext{
From the ${ }^{1}$ Institute for Genome Sciences and Policy, Center for Population Genomics and Pharmacogenetics, Duke University, Durham, North Carolina; ${ }^{2}$ Department of Genetics, University of North Carolina at Chapel Hill, Chapel Hill, North Carolina; ${ }^{3}$ Bioinformatics Research Center, North Carolina State University, Raleigh, North Carolina; ${ }^{4}$ Section of Developmental Pharmacology and Experimental Therapeutics, Children's Mercy Hospitals and Clinics, Kansas City, Missouri; ${ }^{5}$ Department of Psychiatry and Behavioral Sciences, Duke University Medical Center, Durham, North Carolina; and ${ }^{6}$ Department of Biological Psychiatry, John Umstead Hospital, Butner, North Carolina.
}

Iris Grossman, PhD, Pharmacogenetics, GlaxoSmithKline, 5 Moore Drive, Research Triangle Park, NC 27709.E-mail: iris.x.grossman@gsk.com or irisg@isragene.com.

Dr. Goldstein has received unrestricted research support gifts from GlaxoSmithKline and has done consultancy work for Pfizer and Johnson and Johnson.

Dr. Grossman was a consultant for TEVA Pharmaceutical Ltd. Dr Gaedigk is consulting for Osmetech Molecular Diagnostics (Pasadena, CA) and Gentris Corporation (Morrisville, NC).

Dr Gaedigk also serves on the Gentris Scientific Advisory Board.

Dr. Goldstein had full access to all of the data in the study and takes full responsibility for the integrity of the data and the accuracy of the data analysis.

Dr. Sullivan was funded by R01 MH074027 and reports receiving research funding from Eli Lilly. Supplementary material are available via the ArticlePlus feature at www.geneticsinmedicine.org. Submitted for publication May 29, 2008.

Accepted for publication July 9, 2008.

DOI: $10.1097 /$ GIM.0b013e3181863239 variations in DMEs on the pharmacokinetic properties of medications metabolized by these enzymes. ${ }^{3,4}$ This has been particularly true for psychiatric drugs. ${ }^{5-7}$ The above observations have led many to argue that the genotypes of patients at key DME genes should be incorporated into clinical decisionmaking, particularly dosing. ${ }^{7,8}$ As a result, there is an increasing number of companies and products that offer clinicians convenient ways to determine patient genotypes at key DMEs. One product in particular, the Roche AmpliChip, ${ }^{9}$ has been approved by the FDA and is being billed as of specific relevance in the use of a broad range of antipsychotics and antidepressants. Surprisingly, there are few data that clearly support the relevance of DME variation to the decisions that clinicians make in the treatment of either schizophrenia or depression.

The Clinical Antipsychotic Trials of Intervention Effectiveness (CATIE) ${ }^{10}$ assessed the overall effectiveness of five antipsychotics in parallel in a double-blind, randomized fashion. This study design affords an important opportunity to assess how genetic variation among patients may influence clinical decisions. We therefore systematically evaluated the impact of known functional genetic variation in the major and minor DMEs for each tested drug on the doses clinicians decided to prescribe in the CATIE study. Complementary to these analyses, we tested associations between these variants and drugresponse features related to efficacy, safety, and adherence to 
medication. Overall, we found that even validated functional variation in relevant enzymes has but marginal impact on dosing and continued use of medicines. These results suggest that DME-based genotyping tests may be of limited utility in guiding clinicians regarding the optimal use of these medicines in most patients, and that direct assessment of phenotype should be considered as an alternative strategy to optimize drug dosing in this patient population.

\section{MATERIALS AND METHODS}

\section{Subjects}

The study subjects were a subset of patients who had participated in the CATIE study, described at length elsewhere. ${ }^{10}$ Briefly, in Phase 1, patients were randomized to treatment with one of five antipsychotic medications-perphenazine, olanzapine, quetiapine, risperidone, or ziprasidone-and followed for up to 18 months or until treatment discontinuation for any reason. ${ }^{10}$ If perphenazine as the Phase 1 treatment was discontinued, patients entered $P$ hase $1 \mathrm{~B}$ in which they were randomly assigned to receive olanzapine, quetiapine, or risperidone. Discontinuation of Phases 1, 1A, and 1B led to Phase 2, in which randomization pathways were offered ${ }^{11}$ : Phase $2 \mathrm{E}$ compared open-label clozapine treatment to double-blind treatment with olanzapine, quetiapine, or risperidone. Because of the lack of blinding, clozapine is not considered in this report; Phase 2T compared double-blind treatment with olanzapine, quetiapine, risperidone, or ziprasidone. Throughout the complex design of study phases, no subject was randomized to any medication more than once. Patients were not allowed to take other antipsychotics while participating in the trial. However, to mimic real-life settings, concomitant medications for any other indications were not restricted.

Patients were given identically appearing capsules of their assigned drug and instructed to take between one and four pills per day based on the judgment of the treating physician. Each capsule contained $8 \mathrm{mg}$ perphenazine; $7.5 \mathrm{mg}$ olanzapine; 1.5 $\mathrm{mg}$ risperidone; $200 \mathrm{mg}$ quetiapine; or $40 \mathrm{mg}$ ziprasidone.
The study was approved by the institutional review board at each site. Written informed consent was obtained from the patients or their legal guardians. Seven hundred fifty-six study participants consented to provide blood samples for genetic analysis $(52.8 \%$ of patients who had received at least one dose of treatment in CATIE).

\section{Candidate gene selection and hypotheses tested}

All five of the antipsychotics used in CATIE are extensively metabolized into less active or inactive metabolites (Table 1) and some are substrates for transporters active at the bloodbrain barrier such as $\operatorname{PgP}(A B C B 1, M D R 1) .{ }^{12,13}$ The relevant DMEs have been thoroughly screened for functional variation in coding and regulatory genomic regions ${ }^{14}$ and we therefore concentrated our attention on such known polymorphisms. We have considered any known or suspected functional polymorphism with minor allele frequency $>2 \%$ in either whites or African-Americans (Supplementary Materials and Methods Table S1, available online-only), with the exception of CYP2D6 gene duplications.

\section{Genotyping}

All genetic variants, with the exception of the CYP2D6 polymorphisms, were genotyped by TaqMan fluorescence-based allelic discrimination. ${ }^{15}$ Premade assays offered as Assay-byDemand were used when available, otherwise primers were designed using the Applied Biosystems Assay-by-Design tool (Applied Biosystems, Foster City, CA). Polymerase chain reactions were carried out using $0.5 \times$ standard Applied Biosystems protocol for a $5 \mu \mathrm{L}$ reaction volume (Supplementary Materials and Methods Table S2).

CYP2D6 genotyping was performed as described previously, ${ }^{16,17}$ and included ${ }^{*} 2,{ }^{*} 3,{ }^{*} 4,{ }^{*} 5,{ }^{*} 6,{ }^{*} 9,{ }^{*} 10,{ }^{*} 17,{ }^{*} 29$, and ${ }^{\star} 41$. Primer sequences (IDT, Coralville, IA) and additional details pertaining to all genotyping reactions are presented in the Supplementary Materials and Methods Table S2. CYP2D6 allele nomenclature throughout this report uses the recently revised

Table 1

Antipsychotic drugs analyzed and the DMEs that metabolize them

\begin{tabular}{|c|c|c|c|c|c|c|c|c|}
\hline & Drug & Major DME & Secondary DME & $\begin{array}{l}\text { Minor } \\
\text { DME1 }\end{array}$ & Minor DME2 & $\begin{array}{l}\text { Minor } \\
\text { DME3 }\end{array}$ & Transporter & Glucuronidase \\
\hline 1 & Olanzapine & CYP1A2 & FMO3 & CYP2D6 & CYP3A4 and CYP3A5 & & & UGT1A4 \\
\hline 1 & Olanzapine & CYP1A2 & FMO3 & CYP2D6 & CYP3A4 and CYP3A5 & & & UGT1A4 \\
\hline 2 & Quetiapine & CYP3A4 and CYP3A5 & & CYP2D6 & CYP2C9 & & $A B C B 1$ & \\
\hline 3 & Risperidone & CYP2D6 & CYP $3 A 4$ and $C Y P 3 A 5$ & & & & $A B C B 1$ & \\
\hline 4 & Ziprasidone & $A O X 1^{a}$ & CYP $3 A 4$ and CYP3A5 & & & & ABCB1 & \\
\hline 5 & Perphenazine & CYP2D6 & CYP1A2 & CYP2C9 & CYP2C19 & CYP2C8 & & \\
\hline
\end{tabular}

${ }^{a}$ There are no common functional variants in $A O X 1$ and inhibitors and inducers of the enzyme do not affect ziprasidone pharmacokinetics. ${ }^{32,33,40,41} A O X 1$ was thus not analyzed in this report.

DME, drug metabolizing enzyme; CYP, cytochrome P450; $A O X 1$, aldehyde oxidase 1; FMO3, flavin-containing monoxigenase 3; $A B C B 1$, p-glycoprotein (MDR1); UGT1A4, uridine 5' -diphosphate glucuronosyl transferase 1A4. 
allele definitions as established by the CYP2D6 nomenclature committee (http://www.cypalleles.ki.SE/).

\section{Genetic models}

Within each treatment group analyses were performed separately for each DME. The genetic models tested and the scheme by which genotypes were collapsed into phenotypes are described in the Supplementary Materials and Methods (Table S3). Generally, we coded genotype for each gene by the number of functional, active, gene copies present: 2,1 , or 0 . Haplotypes were inferred with PHASE version 2.1 for genes in which two single nucleotide polymorphisms were genotyped (i.e., CYP3A5, UGT1A4, FMO3, ABCB1, CYP2C8, and CYP2C9) to determine the number of active gene copies carried. Analyses were carried out assuming an additive model (power calculations suggest that in situations where roughly $50 \%$ of known causal variants have an additive component, ${ }^{18}$ tests assuming additivity are more powerful than unrestricted genotype tests [unpublished data]).

\section{Phenotype definitions and statistical analyses}

To maximize statistical power, the analyzed independent cohorts included subjects who had received one of the five medications in any treatment phase, given the fact that patients could not receive any specific drug more than once throughout the trial.

\section{Dosing}

Optimized dose was defined as the average of all doses (in capsule number) prescribed from the fourth phase-visit and onward for a specific drug. A visual inspection (by authors DBG and IG) of dose prescription data in a subset of patients indicated frequently fluctuating doses during the first three visits (i.e., a dosage "adjustment" period) followed by relatively stable subsequent regimens. The first doses were therefore excluded from optimized dose definitions, and any patient treated for less than four visits was excluded from the dose analyses. We also considered adherence to medication based on pill-count, self-administered questionnaires and the judgment of CATIE personnel performed each visit and summarized by quartiles reflecting $0-100 \%$ adherence. Patients were included in analyses in phases for which their phase-averaged scores were at least $63 \%$, reflecting overall "usual" (51-75\% of the time) adherence to medication.

Because patients may be noncompliant to medication due to either adverse drug reactions (ADRs) or inadequate efficacy, we tested separately the effect of DME functional variation on adherence to medication as defined above.

Univariate analysis was used to test main effects of each candidate gene as the independent variable versus optimized dose by way of least-squares regression. A multivariate model was constructed using mixed stepwise regression, incorporating the following covariates: age, gender, race (white, black, or other), weight at baseline or phase initiation, maximum weight change throughout the relevant phase, trail phase, phase duration (measured by number of visits), Positive and Negative Syndrome Scale (PANSS) at baseline or phase initiation,
PANSS change throughout the relevant phase, phase-averaged Clinical Global Judgment of Medication Adherence (CGJMA) score, concomitant medications categorized by their inhibitory or inductive effects on the study drugs (summarized in the Supplementary Materials and Methods Table S4), smoking status, occurrence of severe ADRs, tardive dyskinesia (TD) status during the phase, antipsychotic treatment before study initiation, years since first treated with antipsychotics, alcohol, and drug abuse. When more than one enzyme was known to metabolize a study drug the genotype status of the relevant DMEs was incorporated into the model as a potential covariate to account for gene-gene correlations. Criteria for model construction required each covariate to satisfy entry and exit $P$ values of 0.1 or less. Once the covariate model was developed, we compared it to the same model incorporating the relevant genetic effect using the least squares test, as implemented in JMP IN (SAS Institute Inc., Cary NC). ${ }^{19}$ The final model was then tested for gene-by-race interaction and if a significant effect was detected analyses were performed in each ethnic group independently.

\section{Safety and efficacy assessment}

We tested associations between relevant DME gene variants and treatment discontinuation due to safety or inefficacy reasons. These analyses included all patients treated in Phases 1, $1 \mathrm{~A}, 1 \mathrm{~B}, 2 \mathrm{E}$, or $2 \mathrm{~T}$, with the exception of patients discontinuing treatment due to "patient decision" or "subject advocate discontinuation." Additionally, patients discontinuing treatment due to lack of efficacy before the fourth phase-visit were excluded from the efficacy tests. Analysis was performed in a similar manner to that described for the dosing endpoint.

Because of the expectation that poor metabolizers or individuals with reduced DME activity may be at a higher risk to experience dose dependent ADRs and that extensive metabolizers are more likely to show lower efficacy, we constituted two different tests:

1. discontinuation due to ADRs versus full phase completion

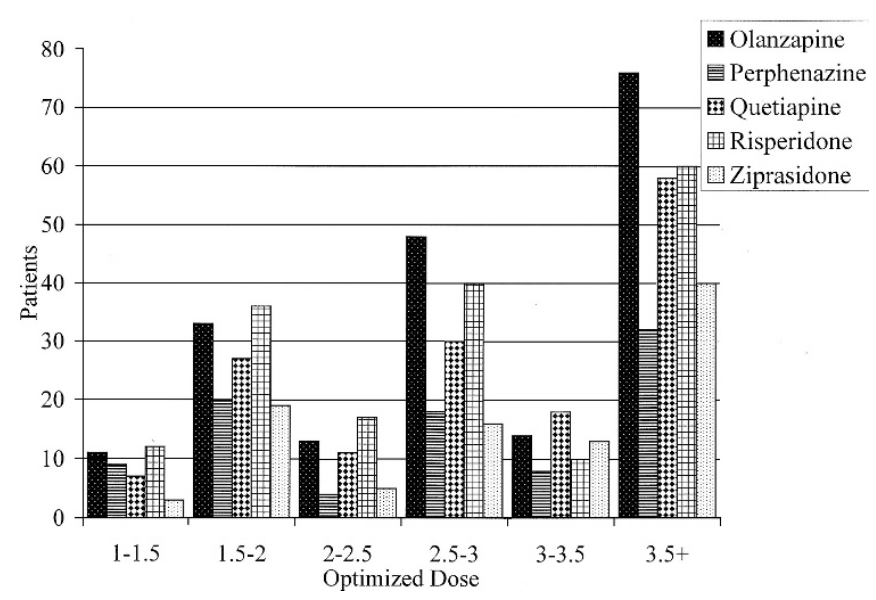

Fig. 1. Histogram displaying the distribution of optimized doses (presented in number of pills taken) in each of the five tested treatment groups. 
Table 2

Description and outcome measures of patients in the optimized dose cohorts

\begin{tabular}{|c|c|c|c|c|c|}
\hline Characteristic & Olanzapine & Quetiapine & Risperidone & Ziprasidone & Perphenazine \\
\hline Patients, OD cohort & 195 & 151 & 175 & 96 & 91 \\
\hline Phase 1/1A & 139 & 104 & 118 & 57 & 91 \\
\hline Phase 1B & 17 & 22 & 12 & 0 & 0 \\
\hline Phase 2 & 39 & 25 & 45 & 39 & 0 \\
\hline Age $(y r)$, mean $\pm S D$ & $40.67 \pm 11.28$ & $41.41 \pm 11.45$ & $40.69 \pm 11.27$ & $40.9 \pm 10.07$ & $40.5 \pm 10.61$ \\
\hline \multicolumn{6}{|l|}{ Sex, no. $(\%)$} \\
\hline Male & $141(72.3)$ & $117(77.5)$ & $131(74.9)$ & $74(77.1)$ & $64(70.3)$ \\
\hline Female & $54(27.7)$ & $34(22.5)$ & $44(25.1)$ & $22(22.9)$ & $27(29.7)$ \\
\hline \multicolumn{6}{|l|}{ Race, no. (\%) } \\
\hline White & $124(63.6)$ & $106(70.2)$ & $117(66.8)$ & $61(63.5)$ & $58(63.7)$ \\
\hline African-American & $63(32.3)$ & $39(25.8)$ & $53(30.3)$ & $29(30.2)$ & $27(29.7)$ \\
\hline Other & $8(4.1)$ & $6(4)$ & $5(2.9)$ & $6(6.3)$ & $6(6.6)$ \\
\hline \multicolumn{6}{|c|}{ BL antipsychotic medications, ${ }^{a}$ no. (\%) } \\
\hline Olanzapine & $71(36.4)$ & $43(28.5)$ & $50(28.6)$ & $23(23.9)$ & $28(30.8)$ \\
\hline Quetiapine & $19(9.7)$ & $11(7.3)$ & $14(8)$ & $10(10.4)$ & $3(3.3)$ \\
\hline Risperidone & $38(19.5)$ & $39(25.8)$ & $42(24)$ & $26(27.1)$ & $26(28.6)$ \\
\hline Perphenazine & 0 & $7(4.6)$ & $5(2.9)$ & $1(1)$ & $1(1.1)$ \\
\hline Ziprasidone & $11(5.6)$ & $8(5.3)$ & $9(5.1)$ & $3(3.1)$ & $2(2.2)$ \\
\hline None & $47(24.1)$ & $35(23.2)$ & $38(21.8)$ & $28(29.2)$ & $25(27.5)$ \\
\hline \multicolumn{6}{|l|}{ Treatment disc, no. (\%) } \\
\hline Ineffectiveness & $25(12.8)$ & $49(32.5)$ & $45(25.7)$ & $23(24)$ & $19(20.9)$ \\
\hline ADRs & $28(14.4)$ & $17(11.3)$ & $15(8.6)$ & $9(9.4)$ & $12(14.3)$ \\
\hline Administrative decision & $8(4.1)$ & $5(3.3)$ & $4(2.3)$ & $4(4.2)$ & $1(1.1)$ \\
\hline Patient decision & $10(5.1)$ & $19^{b}(12.5)$ & $15(8.6)$ & $15(15.6)$ & $7(7.7)$ \\
\hline Treatment completion & $124(63.6)$ & $61(40.4)$ & $96(54.9)$ & $45(46.9)$ & $51(56)$ \\
\hline \multicolumn{6}{|l|}{ TD status, no. (\%) } \\
\hline Probable & $42(21.5)$ & $34(22.5)$ & $30(17.1)$ & $21(21.9)$ & $20(22)$ \\
\hline Definite & $8(4.1)$ & $10(6.6)$ & $6(3.4)$ & $3(3.1)$ & $6(6.6)$ \\
\hline \multicolumn{6}{|l|}{ Weight (lb) } \\
\hline Mean BL & $197.7 \pm 48.33$ & $200.29 \pm 45.54$ & $199.65 \pm 52.67$ & $201 \pm 45.93$ & $191.57 \pm 47.73$ \\
\hline Mean change/mo & $1.05 \pm 1.95$ & $0.28 \pm 3.39$ & $0.26 \pm 2.4$ & $-0.53 \pm 3.13$ & $-0.05 \pm 2.05$ \\
\hline Max phase change & $16.3 \pm 13.79$ & $15.95 \pm 11.88$ & $16.6 \pm 14.2$ & $16.1 \pm 13.04$ & $17.12 \pm 12.76$ \\
\hline \multicolumn{6}{|c|}{ Concomitant medications, no. (\%) } \\
\hline Inhibitors $^{c}$ & $34(17.4)$ & $31(20.5)$ & $28(16)$ & $21(21.9)$ & $14(15.4)$ \\
\hline Inducers $^{c}$ & $12(6.2)$ & $5(3.3)$ & $5(2.9)$ & $3(3.1)$ & - \\
\hline \multicolumn{6}{|l|}{ PANSS score } \\
\hline $\mathrm{BL}$ & $75.10 \pm 19.1$ & $74.1 \pm 16.7$ & $75.7 \pm 16.8$ & $71.1 \pm 14.8$ & $71 \pm 17.6$ \\
\hline Change & $-10.6 \pm 17.04$ & $-3.9 \pm 18.6$ & $-9.4 \pm 18.8$ & $-2.7 \pm 18.9$ & $-8.7 \pm 19.3$ \\
\hline Smoking, no. (\%) & $131(67.2)$ & $105(69.5)$ & $124(70.9)$ & $74(77.1)$ & $66(72.5)$ \\
\hline Cigarettes/day, mean $\pm \mathrm{SD}$ & $12.11 \pm 12.6$ & $10.54 \pm 11.24$ & $12.5 \pm 12$ & $11.92 \pm 11.09$ & $12.9 \pm 12.9$ \\
\hline Alcohol abuse, no. (\%) & $16(8.2)$ & $19(12.6)$ & $19(10.9)$ & $6(6.3)$ & $6(6.6)$ \\
\hline
\end{tabular}


Table 2

Continued

\begin{tabular}{|c|c|c|c|c|c|}
\hline Characteristic & Olanzapine & Quetiapine & Risperidone & Ziprasidone & Perphenazine \\
\hline Drug abuse, no. (\%) & $20(10.3)$ & $20(13.2)$ & $22(12.6)$ & $13(13.5)$ & $12(13.2)$ \\
\hline \multicolumn{6}{|l|}{ No. visits/phase, Mean \pm SD } \\
\hline Phase I/IA & $14.48 \pm 5.15$ & $11.8 \pm 5.44$ & $13.68 \pm 5.25$ & $12.21 \pm 5.52$ & $13.1 \pm 5.6$ \\
\hline Phase IB & $9.88 \pm 3.95$ & $10.77 \pm 3.9$ & $10 \pm 3.54$ & - & - \\
\hline Phase II & $13.35 \pm 5.17$ & $7.12 \pm 3.89$ & $12.27 \pm 5.25$ & $8.23 \pm 3.62$ & - \\
\hline Optimized dose, mean \pm SD & $3.04 \pm 0.88$ & $3.06 \pm 0.88$ & $2.91 \pm 0.9$ & $3.14 \pm 0.86$ & $2.88 \pm 0.96$ \\
\hline $\begin{array}{l}\text { Phase-averaged adherence to medication } \\
\text { score, mean } \pm \text { SD }\end{array}$ & $84.83 \pm 5.43$ & $84.05 \pm 6.12$ & $84.7 \pm 5.37$ & $83.83 \pm 6.15$ & $84.9 \pm 5.55$ \\
\hline Years treated with antipsychotics, mean \pm SD & $14.69 \pm 11.76$ & $13.23 \pm 10.28$ & $13.18 \pm 11.47$ & $13.75 \pm 10.67$ & $13.37 \pm 10.57$ \\
\hline \multicolumn{6}{|l|}{ Anticholinergic treatment, no. (\%) } \\
\hline BL & $42(21.5)$ & $31(20.5)$ & $36(20.6)$ & $19(19.8)$ & $17(18.7)$ \\
\hline During phase & $52(26.7)$ & $29(19.2)$ & $38(21.7)$ & $21(21.9)$ & $25(27.5)$ \\
\hline
\end{tabular}

OD, optimized dose; SD, standard deviation; BL, baseline; disc, discontinuation; ADRs, adverse drug reactions; TD, tardive dyskinesia.

${ }^{a}$ Patients taking any investigational drug within 30 days of the baseline, treatment assignment, visit were excluded.

${ }^{b}$ In one instance the decision to discontinue was made by the patient's advocate.

${ }^{c}$ Concomitant medications were categorized by inhibitor, inducer or no effect on each investigational drug. For details see Supplementary Materials and Methods.

2. discontinuation due to inadequate efficacy versus full phase completion

\section{Tardive dyskinesia}

TD has been suggested to be a dose dependent ADR associated with antipsychotic treatment, particularly "conventional," typical drugs such as perphenazine. TD status was determined based on the Schooler-Kane criteria for probable $\mathrm{TD},{ }^{20}$ for which patients were tested at baseline, every 3 months thereafter, and at phase end. ${ }^{21}$ Analyses were performed with the case group comprised of patients who were positive for the probable TD criteria at least twice throughout their participation in the trial, and the control group including all individuals who never met these criteria. Patients who met the TD criteria only once during the trial were excluded from analyses $(N=74)$. It should be mentioned that while drug treatment immediately before CATIE participation was included in the multivariate analysis (as described in the Dosing section above), we had no access to complete records of medical history.

Analyses were performed by R version 2.3.022 and by JMP IN version $5.1^{19}$ separately and independently within each drug cohort. Hidden population stratification within each ethnicity was not explored in detail. Simple Genomic Control methods were not pursued due to the lack of any significant results. More sophisticated correction methods (e.g., EIGENSTRAT ${ }^{23}$ ) which could correct for false negatives, require an extremely large number of additional genotypes $(>5000)$ to be effective.

\section{RESULTS}

\section{Genotyping results}

All genotype calls were determined independently by at least two researchers and ambiguous calls were re-genotyped or dis- carded. Genotyping failure rates were below 3\%. CYP2C19*3 showed a very low minor allele frequency (as expected) and was excluded from all analyses. Upon testing for Hardy-Weinberg equilibrium, one variant of a total of 16 TaqMan based assays had a $P$-value $<0.05\left(C Y P 2 C 19^{*} 2, P=0.035\right)$, due to a deficiency of heterozygotes. It was re-genotyped, and the same genotypes for all samples were obtained. Allele frequencies were compared with published data and confirmed to match expected values (Supplementary Materials and Methods Table S2). Among the 10 genotyped CYP2D6 variants, Hardy-Weinberg Equilibrium analysis was significant for the ${ }^{\star} 5$ deletion allele (Fisher's exact test $P=0.0016$ ) in whites (of 20 tests in total), the deviation again being due to a deficiency of heterozygotes. The observed rate of significant $P$ values was consistent with that expected under the null hypothesis. These samples, along with 24 randomly picked samples, were re-genotyped independently in a separate blinded laboratory (author AG) and were identically called.

\section{Association results}

Optimized dose

We tested association between optimized dose (Fig. 1) and genotype within each relevant treatment group (Table 2).

None of the tested variants showed a significant main effect $(P<0.05)$ in association with optimized dose in EuropeanAmerican, African-Americans, or all patients (Table 3). Even after accounting for potential covariates no genetic factors were found to be associated with dosing (Table 3).

\section{Safety and efficacy}

Treatment response phenotypes reflecting safety and efficacy were tested by contrasting discontinuation counts due to unacceptable side effects (ADRs) and inadequate efficacy ver- 
Table 3

Association analysis of all relevant DME genetic effects on optimized dose in univariate and covariance analyses

\begin{tabular}{|c|c|c|c|c|}
\hline \multirow[b]{2}{*}{ Drug group } & \multirow[b]{2}{*}{ Gene and variant } & \multicolumn{2}{|c|}{ Main effect } & \multirow{2}{*}{$\begin{array}{c}\text { Analysis of covariance } \\
P\end{array}$} \\
\hline & & Effect size $^{a}$ & $P$ & \\
\hline \multirow[t]{5}{*}{ Perphenazine } & CYP2D6_combined & $\beta=+0.33$ & 0.30 & 0.22 \\
\hline & $C Y P 1 A 2 * 1 F$ & $\beta=+0.17$ & 0.30 & $*$ \\
\hline & CYP2C8_combined & $\beta=-0.33$ & 0.13 & $*$ \\
\hline & CYP2C9_combined & $\beta=-0.08$ & 0.71 & $*$ \\
\hline & CYP2C19*2 & $\beta=-0.001$ & 0.99 & $*$ \\
\hline \multirow[t]{4}{*}{ Risperidone } & CYP2D6_combined & $\beta=-0.07$ & 0.64 & 0.17 \\
\hline & CYP3A5_combined & $\beta=-0.12$ & 0.24 & 0.49 \\
\hline & CYР $3 A 4 * 1 \mathrm{~B}$ & $\beta=-0.06$ & 0.53 & $*$ \\
\hline & $A B C B 1$ _combined & $\beta=-0.12$ & 0.20 & 0.81 \\
\hline \multirow[t]{6}{*}{ Olanzapine } & $C Y P 1 A 2 * 1 F$ & $\beta=-0.15$ & 0.11 & 0.08 \\
\hline & FMO3_combined & $\beta=-0.05$ & 0.56 & 0.54 \\
\hline & UGT1A4_combined & $\beta=+0.07$ & 0.58 & $*$ \\
\hline & CYP3A5_combined & $\beta=-0.002$ & 0.98 & $*$ \\
\hline & $C Y P 1 A 4 * * 1 \mathrm{~B}$ & $\beta=+0.08$ & 0.35 & $*$ \\
\hline & CYP2D6_combined & $\beta=-0.06$ & 0.64 & $*$ \\
\hline \multirow[t]{5}{*}{ Quetiapine } & CYP3A5_combined & $\beta=+0.03$ & 0.79 & 0.46 \\
\hline & СYР $3 A 4 * 1 \mathrm{~B}$ & $\beta=+0.05$ & 0.60 & $*$ \\
\hline & $A B C B 1$ ccombined & $\beta=-0.001$ & 0.99 & $*$ \\
\hline & CYP2D6_combined & $\beta=-0.04$ & 0.78 & $*$ \\
\hline & CYP2C9_combined & $\beta=-0.01$ & 0.94 & $*$ \\
\hline \multirow[t]{3}{*}{ Ziprasidone } & CYP3A5_combined & $\beta=-0.15$ & 0.21 & $0.34^{b}$ \\
\hline & $C Y P 3 A 4 * 1 \mathrm{~B}$ & $\beta=-0.1$ & 0.40 & $*$ \\
\hline & ABCB1_combined & $\beta=+0.02$ & 0.88 & $*$ \\
\hline
\end{tabular}

$\beta$ is the slope of the linear regression model defined by $Y=\alpha+\beta \times X$, where $Y$ is optimized dose and $X$ is the genetic factor tested. Annotation of "gene_combined" relates to the sum functionality of the gene as reflected by the two haplotypes individuals carry (defined in Supplementary Materials and Methods, Table S1). Asterisks (*) denote variants which were considered as potential covariates in the step-wise regression model. Nominal $P$ values are reported.

${ }^{a}$ Main effects are registered in reference to the lower/absent enzymatic activity genotypes, expecting the effect to be positive (i.e., lower activity genotypes are expected to settle on lower optimized doses).

${ }^{b}$ Race-by-gene interaction term was statistically significant $(P=0.04)$ in the ziprasidone cohort, but a multivariate analysis in each race group separately yielded $P=$ 0.36 and 0.12 for African-Americans and whites, respectively.

sus successful phase completion (Table 4), respectively. No nominally significant associations $(P<0.05)$ were detected between the tested variants and occurrence of ADRs in univariate analysis. When exploring potential confounding effects via logistic regression analysis, few nominally significant associations were detected. The only association recorded $(P=0.013)$ in the safety test suggested a correlation between the lower activity variants in the FMO3 gene and higher incidence of ADRs in African-Americans treated with olanzapine $(N=59)$. No association was detected with this variant in either the whole cohort or European-Americans alone. In the efficacy test, we recorded two marginally significant associations between the severely decreased activity genetic variants in CYP3A5 and higher efficacy of quetiapine $(P=0.02)$ and risperidone $(P=0.04)$. None of these results survives correction for multiple testing.

\section{Tardive dyskinesia}

Neither univariate nor regression analyses (Table 5) found the exhibition of TD to be significantly associated $(P<0.05)$ with the lower activity genetic variants of the CYP2D6 and/or CYP1A2 genes.

\section{Medication adherence assessment}

We considered the effect of 23 different drug-DME combinations (Table 1) on two summary indices of medication adherence (the mean of the treating clinician's global judgment scores; and the median proportion of pills taken) using nonparametric and parametric analyses. These two indices showed an inverse correlation (Spearman $\rho=-0.55$ ). None of the tests performed remained significant after adjustment for multiple 
Table 4

Association analysis of DME genetic effects with safety and efficacy phenotypes in each of the tested drug cohorts

\begin{tabular}{|c|c|c|c|c|c|c|c|c|c|c|}
\hline \multirow{2}{*}{$\begin{array}{l}\text { (a) Olanzapine } \\
\text { Genotype }\end{array}$} & \multicolumn{3}{|c|}{$C Y P 1 A 2 * 1 F$} & \multirow{2}{*}{$\begin{array}{c}P \text { (Pearson/Fisher's } \\
\text { exact })\end{array}$} & \multirow{2}{*}{$\begin{array}{c}P(\mathrm{~L}-\mathrm{R} \\
\text { test })\end{array}$} & \multicolumn{3}{|c|}{ FMO3 } & \multirow{2}{*}{$\begin{array}{c}P \text { (Pearson/ Fisher's } \\
\text { exact })\end{array}$} & \multirow{2}{*}{$\begin{array}{l}P(\mathrm{~L}- \\
\text { R test })\end{array}$} \\
\hline & LL & HL & $\mathrm{HH}$ & & & $\mathrm{MM}$ & $\mathrm{MN}$ & $\mathrm{NN}$ & & \\
\hline \multicolumn{11}{|l|}{ Safety test } \\
\hline Disc. Due to ADRs & 5 & 25 & 28 & 0.92 & 0.42 & 16 & 22 & 21 & 0.24 & $0.73^{a}$ \\
\hline Treatment completion & 13 & 52 & 58 & & & 21 & 58 & 45 & & \\
\hline \multicolumn{11}{|l|}{ Efficacy test } \\
\hline Disc. Due to inadequate efficacy & 4 & 6 & 15 & 0.22 & 0.51 & 4 & 13 & 8 & 0.89 & 0.78 \\
\hline Treatment completion & 13 & 52 & 58 & & & 21 & 58 & 45 & & \\
\hline
\end{tabular}

(b) Perphenazine

\begin{tabular}{ccccc}
\multicolumn{3}{c}{ CYP2D6 } & $P($ Pearson/Fisher's & $\begin{array}{c}P(\mathrm{~L}-\mathrm{R} \\
\text { exact })\end{array}$ \\
\cline { 1 - 2 } & IM & EM & &
\end{tabular}

Genotype

Disc. Due to ADRs

$1 \quad 5 \quad 18$

0.13

Treatment completion

$0 \quad 5 \quad 46$

Efficacy test

Disc. Due to inadequate efficacy

$\begin{array}{lll}0 & 0 & 20 \\ 0 & 5 & 46\end{array}$

0.31

0.09

Treatment completion

CYP $3 A 5$

(c) Quetiapine

Genotype

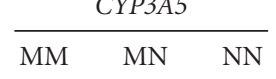

$P$ (Pearson/Fisher's exact)

$P(\mathrm{~L}-\mathrm{R}$

Safety test

Disc. Due to ADRs

$\begin{array}{lll}26 & 11 & 9 \\ 41 & 13 & 8\end{array}$

0.54

0.08

Treatment completion

$33 \quad 11 \quad 5$

0.9

Disc. Due to inadequate efficacy

$\begin{array}{lll}41 & 13 & 8\end{array}$

Treatment completion

\begin{tabular}{ccc}
\multicolumn{3}{c}{ CYP2D6 } \\
\hline PM & IM & EM \\
& & \\
2 & 7 & 27 \\
5 & 7 & 87
\end{tabular}
$P($ Pearson/Fisher's
exact $)$$\quad \begin{gathered}P(\mathrm{~L}-\mathrm{R} \\ \text { test })\end{gathered}$

test)

$\frac{\text { CYP3A5 }}{\mathrm{MM} \quad \mathrm{MN} \quad \mathrm{NN}}$

$P($ Pearson/Fisher's exact)

$P(\mathrm{~L}-$

Genotype

Safety test

Disc. Due to ADRs

Treatment completion

0.08

0.44

24

6

67

$\begin{array}{lll}63 & 28 & 8\end{array}$

Efficacy test

Disc. Due to inadequate efficacy

Treatment completion

$\begin{array}{lll}1 & 3 & 42 \\ 5 & 7 & 87\end{array}$

0.91

0.64

$\begin{array}{lll}24 & 17 & 5 \\ 63 & 28 & 8\end{array}$

(e) Ziprasidone

Genotype

Genotype

\begin{tabular}{lc} 
& \multicolumn{2}{c}{ CYP3A5 } \\
\hline MM & MN
\end{tabular}

LL HL HH

$P$ (Pearson/Fisher's exact)

$P(\mathrm{~L}-\mathrm{R}$ test)

Safety test

Disc. Due to ADRs

$\begin{array}{lll}19 & 8 & 5 \\ 33 & 9 & 5\end{array}$

Treatment completion

Efficacy test

Disc. Due to inadequate efficacy

$\begin{array}{lll}13 & 7 & 3\end{array}$

0.5

0.08

Treatment completion

$33 \quad 9 \quad 5$

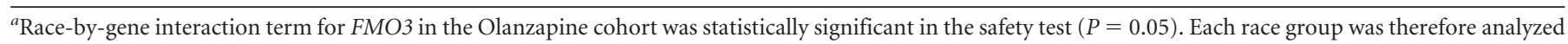
separately, with the African-American group $(\mathrm{N}=59)$ reaching $P=0.013$.

Disc, discontinuation; $M$, mutant/lower activity allele; N, normal/higher activity allele; L, lower inducibility variant; $\mathrm{H}$, higher inducibility allele; L-R, logistic regression. 
DMEs and clinical outcomes in CATIE

Table 5

Association analysis of CYP2D6 phenotype and CYP1A2 genotype with tardive dyskinesia (TD) in univariate analysis and logistic regression (L-R)

\begin{tabular}{|c|c|c|c|c|c|c|c|c|c|c|}
\hline \multirow[b]{2}{*}{ Cohort } & \multirow[b]{2}{*}{ Test } & \multicolumn{3}{|c|}{ CYP2D6 } & \multirow[b]{2}{*}{ Univariate $P$} & \multirow{2}{*}{$\begin{array}{c}\mathrm{L}-\mathrm{R} \text { test } P \\
(\text { racexgene } P \text { ) }\end{array}$} & \multicolumn{3}{|c|}{$C Y P 1 A 2^{\star} 1 \mathrm{~F}$} & \multirow{2}{*}{$\begin{array}{c}\text { Univariate } P \\
\text { (smokers only) }\end{array}$} \\
\hline & & EM & IM & $\mathrm{PM}$ & & & $\mathrm{HH}$ & $\mathrm{LH}$ & LL & \\
\hline \multirow[t]{2}{*}{ All } & TD positive & 113 & 24 & 5 & 0.15 & $0.66(0.1)$ & 65 & 60 & 16 & $0.55(0.45)$ \\
\hline & TD absent & 441 & 59 & 27 & & & 259 & 228 & 45 & \\
\hline \multirow[t]{2}{*}{ White } & TD positive & 71 & 14 & 5 & 0.05 & 0.33 & 44 & 39 & 6 & $0.77(0.41)$ \\
\hline & TD absent & 307 & 26 & 22 & & & 187 & 153 & 18 & \\
\hline \multirow[t]{2}{*}{ African-American } & TD positive & 39 & 7 & 0 & 0.62 & 0.16 & 19 & 17 & 10 & $0.57(0.74)$ \\
\hline & TD absent & 115 & 28 & 4 & & & 59 & 66 & 24 & \\
\hline
\end{tabular}

Pearson's $P$-values are reported for the univariate analysis, accept for CYP2D6 association analysis in African-Americans, where Fisher's exact test $P$-value is listed. EM, extensive metabolizer; IM, intermediate metabolizer; PM, poor metabolizer; H, higher inducibility allele; L, lower inducibility allele.

testing (92 nonindependent tests). The most significant finding observed was for $A B C B 1$ in subjects who had received risperidone: nonparametric $P=0.004$ for clinician judgment based adherence (False Discovery Rate ${ }^{24}(\mathrm{FDR})$-adjusted $\left.P=0.19\right)$ and nonparametric $P=0.04$ for pill count (FDR-adjusted $P=0.36$ ). Even if significance had been achieved, the magnitude of the effect was very small: a median of $96 \%$ of pills taken for the $A B C B 1$ haplotype "0" (coding scheme available in the Supplementary Materials and Methods Table S3) versus $91 \%$ for all other $A B C B 1$ haplotype variants.

\section{DISCUSSION}

Despite the growing enthusiasm for using genetic diagnostics to guide clinical decision-making there remains scant evidence supporting the clinical utility of currently known genetic differences among patients. For DME variants in particular there has been a strong push toward their incorporation into the clinical use of antipsychotics, antidepressants, and other medicines. Here we have evaluated how known functional variation in all the major DMEs for five antipsychotic drugs influences their clinical use in the context of a randomized clinical trial. Despite the relative homogeneity of clinical decision-making in this setting (in comparison, for example, to care outside of a trial setting) and a comprehensive set of clinical and genetic covariates tested we find no strong associations between the relevant genetic variants and the safety or efficacy of the medicines or even with their optimized doses. These results do not, of course, rule out modest effects or potential impact of gene-gene and gene-environment synergies not well understood yet, that may overall be clinically meaningful. Power analyses ${ }^{25}$ suggest, however, that our study was wellpowered to detect minimum genetic effects of between $6 \%$ and $20 \%$ of variance in the dosing phenotype, as well as minimum genotype relative risks of between 1.6 and 2.7 for the discontinuation tests. This suggests that in normal clinical settings DMEs variation has, at best, modest effects on the use of these medicines.

A few inherent limitations in the current study should be noted. First, pharmacogenetic variability in drug biotransfor- mation is most likely to have clinical consequence when the clearance of the drug is primarily or solely dependent on a single pathway. Although CYP2D6 is the lead determinant of perphenazine clearance, alternative pathways may play important roles (Table 1). As patients differ in their individual complement of hepatic and intestinal CYPs, the clinical impact of CYP2D6 genotype and phenotype will vary depending on the relative importance of CYP2D6- versus non-CYP2D6-mediated clearance pathways. For example, when expressed relative to the administered dose, median steady state concentrations of perphenazine were almost double in CYP2D6 poor metabolizers compared with extensive metabolizers, but overlapped in range. ${ }^{26}$ Second, risperidone and its CYP2D6-dependent metabolite, 9-hydroxyrisperidone, are considered to have similar pharmacologic activity ${ }^{27}$ and are jointly referred to as the active moiety. Although a relationship between CYP2D6 genotype and phenotypes, such as steady state serum risperidone concentration/dose ratios and risperidone/9-hydroxyrisperidone ratios, has been established by several groups and has potentially been associated with $\mathrm{ADRs},{ }^{28}$ there appears to be no such relationship between genotype and total active moiety. ${ }^{29}$ On the other hand, any pathway affecting the latter (e.g., $\mathrm{N}$-dealkylation by CYP $3 A,{ }^{27}$ Table 1) potentially confers prognostic value. Third, the use of genotype data to predict drug clearance and dose requirements or toxicity is particularly difficult when enzyme activity demonstrates considerable variability in a particular population and is inducible. For example, phenotypic differences in CYP1A2 activity are more apparent in smokers ${ }^{30}$ (compelling us to perform analyses conditioned on smoking status and consider smoking as a covariate in multiple regression models). However, it is unclear what is the quantitative importance of the polymorphic pathway in the overall disposition of a drug of interest. ${ }^{31}$ Fourth, interpretation and application of genotype information is also compounded by knowledge of the likely functional consequences of the genotyped single nucleotide polymorphism (or variants in linkage equilibrium) on the specific drugs of interest in specific populations. One example is the $C Y P 2 D 6^{\star} 17$ allele, which demonstrates "reduced" activity toward dextromethorphan, bufuralol, and debrisoquine, relative to the reference $C Y P 2 D 6^{*} 1$ alleles, but has 
been reported to convey increased activity toward other medications, such as haloperidol ${ }^{32}$ and risperidone ${ }^{33}$ in specific populations. Last, recently published data indicates the potential of variants not explored in the current report to affect drug response phenotypes, such as the recently reported CYP2C19* $17 .{ }^{34}$ The above phenomena, if extends to other CYPs and substrates, may further obfuscate genotype-phenotype relationships.

Reviewing the above articulated considerations, evidence for clinical utility of routine genotyping of genes involved in drug biotransformation and transport likely will first be limited to situations where a single, polymorphically expressed pathway is the primary route of elimination in most treated patients, the drug is metabolized to inactive metabolites and has a narrow range. More direct measurement of drug biotransformation phenotype, as supplements to our estimations from genotype data, is currently being evaluated in a subset of the CATIE subjects.

In summary, our results suggest that genotype information alone is a poor predictor of antipsychotic drug disposition and response in a clinical situation. Caution is warranted when using diagnostic products such as the Roche AmpliChip in absence of a clear association between the tested variants and relevant clinical responses. More generally, taken together with the report of no significant association between functional DME variants and citalopram response in the Sequenced Treatment Alternatives to Relieve Depression $\left(\mathrm{STAR}^{\star} \mathrm{D}\right)$ tri$\mathrm{al}^{35}$ these results may suggest that the importance of genetically controlled variability in drug pharmacokinetics may often be modest. Although improvements in prediction of phenotypes by usage of genotype information are constantly being refined, ${ }^{36,37}$ prospective data demonstrating the utility of a priori DME genotype assessment for optimizing treatment response are extremely limited. In this context, it should be mentioned that variation in genes related to a medication's mode of action may often have larger effects than variants in genes influencing metabolism, ${ }^{38,39}$ though it is too early to be certain how general this will prove. What does appear likely is that if variation in genes governing the pharmacodynamic properties of drugs does not prove to be more important than those governing pharmacokinetics, germline pharmacogenetics will have at best a very modest clinical utility in many therapeutic areas, and specifically in psychiatry.

\section{ACKNOWLEDGMENTS}

We thank the CATIE subjects for their participation in this investigation and the CATIE executive committee for its guidance.

The CATIE study was supported by NIMH contract N01 MH90001 (PI J Lieberman).

\section{References}

1. Eichelbaum M. Polymorphic drug oxidation in humans. Fed Proc 1984;43: 2298-2302.

2. Hesselink DA, van Schaik RH, van der Heiden IP, et al. Genetic polymorphisms of the CYP3A4, CYP3A5, and MDR-1 genes and pharmacokinetics of the calcineurin inhibitors cyclosporine and tacrolimus. Clin Pharmacol Ther 2003;74:245-254.
3. Gaedigk A, Gotschall RR, Forbes NS, Simon SD, Kearns GL, Leeder JS. Optimization of cytochrome P4502D6 (CYP2D6) phenotype assignment using a genotyping algorithm based on allele frequency data. Pharmacogenetics 1999;9:669-682.

4. Meyer UA, Zanger UM. Molecular mechanisms of genetic polymorphisms of drug metabolism. Annu Rev Pharmacol Toxicol 1997;37:269-296.

5. Arranz MJ, de Leon J. Pharmacogenetics and pharmacogenomics of schizophrenia: a review of last decade of research. Mol Psychiatry 2007;12:707-747.

6. Dahl ML. Cytochrome 450 phenotyping/genotyping in patients receiving antipsychotics: useful aid to prescribing? Clin Pharmacokinet 2002;41:453-470.

7. Kirchheiner J, Nickchen K, Bauer M, et al. Pharmacogenetics of antidepressants and antipsychotics: the contribution of allelic variations to the phenotype of drug response. Mol Psychiatry 2004;9:442-473.

8. de Leon J, Armstrong SC, Cozza KL. The dosing of atypical antipsychotics. Psychosomatics 2005;46:262-273.

9. de Leon J. AmpliChip CYP450 test: personalized medicine has arrived in psychiatry. Expert Rev Mol Diagn 2006;6:277-286.

10. Lieberman JA, Stroup TS, McEvoy JP, et al. Effectiveness of antipsychotic drugs in patients with chronic schizophrenia. N Engl J Med 2005;353:1209-1223.

11. Stroup TS, Lieberman JA, McEvoy JP, et al. Effectiveness of olanzapine, quetiapine, risperidone, and ziprasidone in patients with chronic schizophrenia following discontinuation of a previous atypical antipsychotic. Am J Psychiatry 2006;163:611-622.

12. Markowitz JS, DeVane CL, Liston HL, Boulton DW, Risch SC. The effects of probenecid on the disposition of risperidone and olanzapine in healthy volunteers. Clin Pharmacol Ther 2002;71:30-38.

13. Wang JS, Ruan Y, Taylor RM, Donovan JL, Markowitz JS, DeVane CL. The brain entry of risperidone and 9-hydroxyrisperidone is greatly limited by P-glycoprotein. Int J Neuropsychopharmacol 2004;7:415-419.

14. Sim SC, Ingelman-Sundberg M. The human cytochrome P450 allele nomenclature committee web site: submission criteria, procedures, and objectives. Methods Mol Biol 2006;320:183-191.

15. Livak KJ. Allelic discrimination using fluorogenic probes and the $5^{\prime}$ nuclease assay. Genet Anal 1999;14:143-149.

16. Gaedigk A, Gotschall RR, Forbes NS, Simon SD, Kearns GL, Leeder JS. Optimization of cytochrome P4502D6 (CYP2D6) phenotype assignment using a genotyping algorithm based on allele frequency data. Pharmacogenetics 1999;9:669-682.

17. Gaedigk A, Bradford LD, Marcucci KA, Leeder JS. Unique CYP2D6 activity distribution and genotype-phenotype discordance in black Americans. Clin Pharmacol Ther 2002;72:76-89.

18. Hirschhorn JN, Lohmueller K, Byrne E, Hirschhorn K. A comprehensive review of genetic association studies. Genet Med 2002;4:45-61.

19. Sall J, Ann L, Lee C. JMP start statistics. Pacific Grove, CA: Duxbury Press, 2001.

20. Schooler NR, Kane JM. Research diagnoses for tardive dyskinesia. Arch Gen Psychiatry 1982;39:486-487.

21. Miller DD, McEvoy JP, Davis SM, et al. Clinical correlates of tardive dyskinesia in schizophrenia: baseline data from the CATIE schizophrenia trial. Schizophr Res 2005;80:33-43.

22. R Development Core Team. R:a language and environment for statistical computing. Vienna, Austria: R Foundation for Statistical Computing, 2006.

23. Price AL, Patterson NJ, Plenge RM, Weinblatt ME, Shadick NA, Reich D. Principal components analysis corrects for stratification in genome-wide association studies. Nat Genet 2006;38:904-909.

24. Benjamini Y, Hochberg Y. Controlling the false discovery rate: a practical and powerful approach to multiple testing. J Royal Stat Soc Ser B1995;57:289-300.

25. Purcell S, Cherny SS, Sham PC. Genetic power calculator: design of linkage and association genetic mapping studies of complex traits. Bioinformatics 2003;19: $149-150$.

26. Linnet K, Wiborg O. Steady-state serum concentrations of the neuroleptic perphenazine in relation to CYP2D6 genetic polymorphism. Clin Pharmacol Ther 1996; 60:41-47.

27. Huang ML, Van Peer A, Woestenborghs R, et al. Pharmacokinetics of the novel antipsychotic agent risperidone and the prolactin response in healthy subjects. Clin Pharmacol Ther 1993;54:257-268.

28. de Leon J, Susce MT, Pan RM, Fairchild M, Koch WH, Wedlund PJ. The CYP2D6 poor metabolizer phenotype may be associated with risperidone adverse drug reactions and discontinuation. J Clin Psychiatry 2005;66:15-27.

29. Scordo MG, Spina E, Facciola G, Avenoso A, Johansson I, Dahl ML. Cytochrome P450 2D6 genotype and steady state plasma levels of risperidone and 9-hydroxyrisperidone. Psychopharmacology (Berl) 1999;147:300-305.

30. Sachse C, Brockmoller J, Bauer S, Roots I. Functional significance of a C->A polymorphism in intron 1 of the cytochrome P450 CYP1A2 gene tested with caffeine. Br J Clin Pharmacol 1999;47:445-449.

31. van der WJ, Steijns LS, van Weelden MJ. The effect of smoking and cytochrome 
P450 CYP1A2 genetic polymorphism on clozapine clearance and dose requirement. Pharmacogenetics 2003;13:169-172.

32. Bogni A, Monshouwer M, Moscone A, et al. Substrate specific metabolism by polymorphic cytochrome P450 2D6 alleles. Toxicol In Vitro 2005;19:621-629.

33. Cai WM, Nikoloff DM, Pan RM, et al. CYP2D6 genetic variation in healthy adults and psychiatric African-American subjects: implications for clinical practice and genetic testing. Pharmacogenomics J 2006;6:343-350.

34. Rudberg I, Mohebi B, Hermann M, Refsum H, Molden E. Impact of the ultrarapid CYP2C19* 17 allele on serum concentration of escitalopram in psychiatric patients. Clin Pharmacol Ther 2008;83:322-327.

35. Peters EJ, Slager SL, Kraft JB, et al. Pharmacokinetic genes do not influence response or tolerance to citalopram in the STAR ${ }^{\star}$ D sample. PLoS ONE 2008;3: e1872.

36. Schenk PW, van Fessem MA, Verploegh-Van Rij S, et al. Association of graded allele-specific changes in CYP2D6 function with imipramine dose requirement in a large group of depressed patients. Mol Psychiatry 2008;13:597-605.
37. Gaedigk A, Simon SD, Pearce RE, Bradford LD, Kennedy MJ, Leeder JS. The CYP2D6 activity score: translating genotype information into a quantitative measure of phenotype. Clin Pharmacol Ther 2008;83:234-242.

38. Aquilante CL, Langaee TY, Lopez LM, et al. Influence of coagulation factor, vitamin K epoxide reductase complex subunit 1, and cytochrome P450 2C9 gene polymorphisms on warfarin dose requirements. Clin Pharmacol Ther 2006;79: 291-302.

39. Tate SK, Depondt C, Sisodiya SM, et al. Genetic predictors of the maximum doses patients receive during clinical use of the anti-epileptic drugs carbamazepine and phenytoin. Proc Natl Acad Sci USA 2005;102:5507-5512.

40. Beedham C, Miceli JJ, Obach RS. Ziprasidone metabolism, aldehyde oxidase, and clinical implications. J Clin Psychopharmacol 2003;23:229-232.

41. Obach RS, Walsky RL. Drugs that inhibit oxidation reactions catalyzed by aldehyde oxidase do not inhibit the reductive metabolism of ziprasidone to its major metabolite, S-methyldihydroziprasidone: an in vitro study. J Clin Psychopharmacol 2005; 25:605-608. 